\title{
Discrete-frequency Convergence of Iterative Learning Control for Linear Time-invariant systems with Higher-order Relative Degree
}

\author{
Xiao-E Ruan ${ }^{1} \quad$ Zhao-Zhen $\mathrm{Li}^{1} \quad$ Z. Z. Bien ${ }^{2}$ \\ ${ }^{1}$ School of Mathematics and Statistics, $\mathrm{Xi}^{\prime}$ an Jiaotong University, Xi' an 710049, China \\ ${ }^{2}$ Department of Electrical Engineering, Korea Advanced Institute of Science and Technology, Daejeon, 305-701, Korea
}

\begin{abstract}
In this paper, a discrete-frequency technique is developed for analyzing sufficiency and necessity of monotone convergence of a proportional higher-order-derivative iterative learning control scheme for a class of linear time-invariant systems with higher-order relative degree. The technique composes of two steps. The first step is to expand the iterative control signals, its driven outputs and the relevant signals as complex-form Fourier series and then to deduce the properties of the Fourier coefficients. The second step is to analyze the sufficiency and necessity of monotone convergence of the proposed proportional higher-order-derivative iterative learning control scheme by assessing the tracking errors in the forms of Paserval's energy modes. Numerical simulations are illustrated to exhibit the validity and the effectiveness.
\end{abstract}

Keywords: Iterative learning control, monotone convergence, discrete frequency-domain spectrum, Fourier series, Parseval's energy equality, relative degree.

\section{Introduction}

As one of intelligent control methodologies, the concept of iterative learning control (ILC) was proposed by Arimoto in 1980s for a robot manipulator to track a desired trajectory while it attempts to execute a sequence of repetitive tasks over a fixed time interval ${ }^{[1]}$. The fundamental mechanism of the ILC is to utilize the proportional, integral and/or derivative tracking error(s) at the current iteration to compensate for its control input so as to iteratively generate the control input for the next iteration. The aim is to achieve that the constructed iterative control inputs drive the system to track the desired trajectory as precisely as possible as the iteration index goes to infinity. A number of ILC investigations have been emerged in favor of less system information requirement and distinct algorithmic structure ${ }^{[2-6]}$.

In the ILC community, one of the involved concepts is the relative degree of the system, which describes the grade of the system control input to directly feed the output bridged by the system dynamics such as the system matrix, the input matrix as well as the output matrix. Regarding the ILC investigation for the system with a higher-order relative degree, Sun et al. ${ }^{[7]}$ proposed a learning algorithm with initial rectifying action for a class of nonlinear systems

Regular Paper

Special Issue on Latest Advances in ILC/RLC Theory and Applications

Manuscript received April 29, 2014; accepted October 28, 2014

This work was supported by National Natural Science Foundation of China (Nos. F010114-60974140 and 61273135)

Recommended by Guest Editor Rong-Hu Chi

(C) Institute of Automation, Chinese Academy of Science and Springer-Verlag Berlin Heidelberg 2015 with higher-order relative degree. Song et al. ${ }^{[8]}$ proposed a first-order derivative-type (D-type) ILC based on a dummy model for nonlinear system with unknown relative degree. Meanwhile, the sampled-data ILC and the anticipatory ILC methods have been developed for single-input-single-output (SISO) nonlinear system with arbitrary relative degree ${ }^{[9,10]}$. Recently, Ruan et al. ${ }^{[1]}$ investigated the monotone convergence of the $r$-th-order derivative-type $\left(\mathrm{D}^{(r)}\right.$-type) ILC scheme for linear time-invariant systems with a higher relative degree $r$ being larger than identity, in which the tracking error is measured in the form of Lebesgue- $p$ norm ${ }^{[11]}$. The investigations convey that efficiency achieved by the derivative-type ILC law mainly hinges on the wellness of the order in terms of the derivative in the ILC algorithm which matches the system relative degree. However, the above-mentioned contributions involve only the sufficient conditions for convergence. As the necessity for convergence is illuminant and beneficial, it is inspiring to exploit any sufficient and necessary condition for monotone convergence.

From an engineering point of view, the frequency domain technique is sometimes preferable as it may exhibit the spectrum feature of a signal and may take advantage of its lower computation complexity in a multiplication form of the transfer functions converted by Laplace transform ${ }^{[12-14]}$. Usually in engineering, the spectrum function $F(\mathrm{j} \omega)$ of a signal $f(t)$ for all $t \geq 0$ is induced directly from its Laplace transform defined as $F(s)=L(f(t))=\int_{0}^{+\infty} f(t) \mathrm{e}^{-s t} \mathrm{~d} t$ by setting the real part of the complex variable $s=\sigma+\mathrm{j} \omega$ to be zero, i.e., $\operatorname{Re}(s)=\sigma=0$. This implies that the spectrum function $F(\mathrm{j} \omega)$ is obtained on the premise that the Laplace integral $F(s)$ is existent for the case when $\operatorname{Re}(s)=0$. But, 
mathematically, for some functions, the existence of the abnormal type of Laplace integral $F(s)$ requires the real part of the complex variable $s=\sigma+\mathrm{j} \omega$ to be no less than a positive constant. In addition, as a frequency domain technique, Laplace transform is fit for the system operating over the whole time duration from zero to infinity and thus the spectrum function is frequency-continuous. Note that the ILC scheme is implementable for a system with multiple operations, where each operation is performed over a finite time interval. This implies that the Laplace-transform spectrumbased ILC results reported in [12-14] need to be refined in a rigorous manner. However, Dirichlet has exploited a discrete-frequency technique by which a Dirichlet-type signal over a finite time interval is expanded as a Fourier series. This makes it possible to adopt Fourier series for enlightening the ILC significance. The aforementioned expectations motivate the paper to investigate sufficient and necessary assumptions in discrete frequency domain for monotone convergence of a proportional-higher-order-derivativetype iterative learning control $\left(\mathrm{PD}^{(r)}\right.$-type ILC) algorithm for a class of linear time-invariant system with a relative degree $r>1$.

The remaining of the paper is organized as follows. Section 2 exhibits preliminaries including the concept of the system relative degree, the well-known Dirichlet theorem, some relevant properties of the Fourier coefficients and the discrete frequency-domain Parseval's energy equality. Section 3 constructs a $\mathrm{PD}^{(r)}$-type ILC scheme and derives the Fourier coefficients of the tracking errors. In Section 4, sufficiently and necessarily monotonous convergence of the proposed $\mathrm{PD}^{(r)}$-type ILC algorithm is analyzed in discrete frequency domain. Numerical simulations are displayed in Section 5, and Section 6 concludes the paper.

\section{Preliminaries}

Consider a class of single-input-single-output linear timeinvariant systems described as

$$
\left\{\begin{array}{l}
\dot{x}(t)=A x(t)+B u(t) \\
y(t)=C x(t), t \in[0, T]
\end{array}\right.
$$

where $[0, T]$ is the time interval of system operation, $x(t) \in$ $\mathbf{R}^{n}, u(t) \in \mathbf{R}$ and $y(t) \in \mathbf{R}$ denote the $n$-dimensional state vector, scalar control input and output, respectively. $A, B$ and $C$ are matrices with appropriate dimensions.

Definition $\mathbf{1}^{[15]}$. Suppose that the system (1) satisfies the following conditions:

$$
\left\{\begin{array}{l}
C A^{i-1} B=0, i=1,2, \cdots, r-1 \\
C A^{r-1} B \neq 0 .
\end{array}\right.
$$

Then, the relative degree of system (1) is said to be $r$, where $r$ is an integer larger than one and $A^{i}(i=0,1, \cdots, r-$ 1) represents the $i$-th power of matrix $A$.

For the system dynamics (1), the following derivation is helpful in understanding the concept of the system relative degree.
If the relative degree $r=1$, then $C B \neq 0$. Thus, it yields

$$
\begin{aligned}
& y(t)=C x(t) \\
& \dot{y}(t)=C \dot{x}(t)=C A x(t)+C B u(t) .
\end{aligned}
$$

If the relative degree $r=2$, it implies that $C B=0$ and $C A B \neq 0$. Therefore,

$$
\begin{aligned}
& \dot{y}(t)=C A x(t) \\
& \ddot{y}(t)=\frac{\mathrm{d}(\dot{y}(t))}{\mathrm{d} t}=C A \dot{x}(t)=C A(A x(t)+B u(t))= \\
& C A^{2} x(t)+C A B u(t) .
\end{aligned}
$$

Likewise, if the relative degree $r=3$ which indicates $C A B=0$ and $C A^{2} B \neq 0$, then $\ddot{y}(t)=C A^{2} x(t)$ which gives rise to

$$
\begin{gathered}
\dddot{y}(t)=C A^{2} \dot{x}(t)=C A^{2}(A x(t)+B u(t))= \\
C A^{3} x(t)+C A^{2} B u(t) .
\end{gathered}
$$

In general, if the relative degree of the system (1) is $r>1$, we get

$$
\left\{\begin{array}{l}
y^{(i)}(t)=C A^{i} x(t), 0 \leq i \leq r-1 \\
y^{(r)}(t)=C A^{r} x(t)+C A^{r-1} B u(t) .
\end{array}\right.
$$

From equation (3), it is seen that the relative degree $r$ turns to be the lowest derivative order of the output $y(t)$ whose lowest-order derivative $y^{(r)}(t)$ is explicitly fed by the control input $u(t)$ bridged by the system matrices.

Dirichlet Theorem ${ }^{[16]}$. If a periodic function $f(t), t \in$ $(-\infty,+\infty)$ with a period $T$ is piecewise monotone on the interval $[0, T]$ and is continuous except possibly for a finite number of discontinuous points of the first type, then the function $f(t)$ can be decomposed as a Fourier series in a complex form as

$$
S(t)=\sum_{n=-\infty}^{+\infty} C_{n} \mathrm{e}^{\mathrm{j} n \omega t}
$$

where

$$
\begin{aligned}
& \omega=\frac{2 \pi}{T}, \quad \mathrm{j}^{2}=-1, \quad \mathrm{e}^{\mathrm{j} n \omega t}=\cos (n \omega t)+\mathrm{j} \sin (n \omega t) \\
& C_{0}=\frac{1}{T} \int_{0}^{T} f(t) \mathrm{d} t, \quad C_{n}=\frac{1}{T} \int_{0}^{T} f(t) \mathrm{e}^{-\mathrm{j} n \omega t} \mathrm{~d} t \\
& n=0, \pm 1, \pm 2, \cdots \\
& S(t)=\left\{\begin{array}{c}
f(t), \quad \text { if } \quad t \text { is a point of continuity, } \\
\frac{1}{2}\left[\lim _{\Delta t \rightarrow 0^{+}} f(t+\Delta t)+\lim _{\Delta t \rightarrow 0^{-}} f(t+\Delta t)\right], \\
\text { if } t \text { is a point of discontinuity, } \\
\frac{1}{2}\left[\lim _{\Delta t \rightarrow 0^{+}} f(0+\Delta t)+\lim _{\Delta t \rightarrow 0^{-}} f(T+\Delta t)\right], \\
\text { if } t=0 \quad \text { or } T .
\end{array}\right.
\end{aligned}
$$

In the summation (4), the terms $\sin (\omega t)$ and $\cos (\omega t)$ produced by $C_{-1} \mathrm{e}^{-\mathrm{j} \omega t}+C_{1} \mathrm{e}^{\mathrm{j} \omega t}$ are called fundamental sinusoidal and cosine waves, whilst the terms $\sin (n \omega t)$ and $\cos (n \omega t)$ produced by $C_{-n} \mathrm{e}^{-\mathrm{j} n \omega t}+C_{+n} \mathrm{e}^{\mathrm{j} n \omega t}$ for $n=$ 
$2,3, \cdots$, are named as higher-frequency harmonic waves. Usually, the summation $S(t)=\sum_{n=-\infty}^{+\infty} C_{n} \mathrm{e}^{\mathrm{j} n \omega t}$ is called as a Fourier series expansion of the function $f(t)$ and denoted by $f(t)=\sum_{n=-\infty}^{+\infty} C_{n} \mathrm{e}^{\mathrm{j} n \omega t}$ for convenience. The above function $f(t)$ is called as a Dirichlet-type function.

Denote the Fourier coefficient $C_{n}$ of the function $f(t)$ as $F(n \omega)$, i.e.,

$$
F(n \omega)=C_{n}=\frac{1}{T} \int_{0}^{T} f(t) \mathrm{e}^{-\mathrm{j} n \omega t} \mathrm{~d} t, \quad n=0, \pm 1, \pm 2, \cdots
$$

Then,

$$
f(t)=\sum_{n=-\infty}^{+\infty} F(n \omega) \mathrm{e}^{\mathrm{j} n \omega t}
$$

Thus, $F(n \omega)$ and $f(t)$ can be regarded as a Fourier pair.

For the sake of simplifying the context statement, in following content, all of the formulations in terms of discrete frequency " $n \omega$ " represent that they are fit for all frequency orders, $n=0, \pm 1, \pm 2, \cdots$.

Lemma 1.

Property 1. If $F_{1}(n \omega)=\frac{1}{T} \int_{0}^{T} f_{1}(t) \mathrm{e}^{-\mathrm{j} n \omega t} \mathrm{~d} t$ and $F_{2}(n \omega)=\frac{1}{T} \int_{0}^{T} f_{2}(t) \mathrm{e}^{-\mathrm{j} n \omega t} \mathrm{~d} t$, then $\alpha F_{1}(n \omega)+\beta F_{2}(n \omega)=$ $\frac{1}{T} \int_{0}^{T}\left(\alpha f_{1}(t)+\beta f_{2}(t)\right) \mathrm{e}^{-\mathrm{j} n \omega t} \mathrm{~d} t$,

$n=0, \pm 1, \pm 2, \cdots, \alpha$ and $\beta$ are constants.

\section{Property 2.}

$$
\begin{aligned}
& F^{(r)}(n \omega)=\frac{1}{T} \int_{0}^{T} f^{(r)}(t) \mathrm{e}^{-\mathrm{j} n \omega t} \mathrm{~d} t= \\
& (\mathrm{j} n \omega)^{r} F(n \omega)+\frac{1}{T} \sum_{i=0}^{r-1}(\mathrm{j} n \omega)^{r-1-i}\left(f^{(i)}(T)-f^{(i)}(0)\right), \\
& r=1,2,3, \cdots .
\end{aligned}
$$

Proof. If $r=1$, then we get

$$
\begin{aligned}
& F^{(1)}(n \omega)=\frac{1}{T} \int_{0}^{T} \frac{\mathrm{d} f(t)}{\mathrm{d} t} \mathrm{e}^{-\mathrm{j} n \omega t} \mathrm{~d} t= \\
& \frac{1}{T}\left[\left.f(t) \mathrm{e}^{-\mathrm{j} n \omega t}\right|_{0} ^{T}+\mathrm{j} n \omega \int_{0}^{T} f(t) \mathrm{e}^{-\mathrm{j} n \omega t} \mathrm{~d} t\right]= \\
& \frac{1}{T}\left[(f(T)-f(0))+\mathrm{j} n \omega \int_{0}^{T} f(t) \mathrm{e}^{-\mathrm{j} n \omega t} \mathrm{~d} t\right]= \\
& \frac{1}{T}(f(T)-f(0))+\mathrm{j} n \omega F(n \omega) .
\end{aligned}
$$

If $r=2$, then we get

$$
\begin{aligned}
& F^{(2)}(n \omega)=\frac{1}{T} \int_{0}^{T} f^{\prime \prime}(t) \mathrm{e}^{-\mathrm{j} n \omega t} \mathrm{~d} t= \\
& \mathrm{j} n \omega F^{(1)}(n \omega)+\frac{1}{T}\left(f^{\prime}(T)-f^{\prime}(0)\right)= \\
& (\mathrm{j} n \omega)^{2} F(n \omega)+\mathrm{j} n \omega \frac{1}{T}(f(T)-f(0))+ \\
& \frac{1}{T}\left(f^{\prime}(T)-f^{\prime}(0)\right) .
\end{aligned}
$$

Again, if $r=3$, then

$$
\begin{aligned}
& F^{(3)}(n \omega)=\frac{1}{T} \int_{0}^{T} f^{(3)}(t) \mathrm{e}^{-\mathrm{j} n \omega t} \mathrm{~d} t= \\
& \mathrm{j} n \omega F^{(2)}(n \omega)+\frac{1}{T}\left(f^{\prime \prime}(T)-f^{\prime \prime}(0)\right)= \\
& (\mathrm{j} n \omega)^{3} F(n \omega)+(j n \omega)^{2} \frac{1}{T}(f(T)-f(0))+ \\
& \mathrm{j} n \omega \frac{1}{T}\left(f^{\prime}(T)-f^{\prime}(0)\right)+\frac{1}{T}\left(f^{\prime \prime}(T)-f^{\prime \prime}(0)\right) .
\end{aligned}
$$

Analogously,

$$
\begin{gathered}
F^{(r)}(n \omega)=\frac{1}{T} \int_{0}^{T} f^{(r)}(t) \mathrm{e}^{-\mathrm{j} n \omega t} \mathrm{~d} t=(\mathrm{j} n \omega)^{r} F(n \omega)+ \\
\frac{1}{T} \sum_{i=0}^{r-1}(\mathrm{j} n \omega)^{r-1-i}\left(f^{(i)}(T)-f^{(i)}(0)\right)
\end{gathered}
$$

Property 3. $|F(-n \omega)|=|F(n \omega)|$.

Proof.

$$
\begin{gathered}
|F(-n \omega)|=\left|\frac{1}{T} \int_{0}^{T} f(t) \mathrm{e}^{-\mathrm{j}(-n \omega) t} \mathrm{~d} t\right|= \\
\left|\frac{1}{T} \int_{0}^{T} f(t) \overline{\mathrm{e}^{-\mathrm{j} n \omega t} \mathrm{~d} t}\right|=\left|\frac{1}{T} \overline{\int_{0}^{T} f(t) \mathrm{e}^{-\mathrm{j} n \omega t} \mathrm{~d} t}\right|= \\
|\overline{F(n \omega)}|=|F(n \omega)| .
\end{gathered}
$$

\section{Property 4.}

$$
\frac{1}{T} \int_{0}^{T} f_{1}(t) f_{2}(t) \mathrm{d} t=\sum_{n=-\infty}^{+\infty} F_{1}(n \omega) F_{2}(-n \omega) .
$$

\section{Proof.}

$$
\begin{aligned}
\frac{1}{T} \int_{0}^{T} & f_{1}(t) f_{2}(t) \mathrm{d} t= \\
& \frac{1}{T} \int_{0}^{T} f_{2}(t) \sum_{n=-\infty}^{+\infty} F_{1}(n \omega) \mathrm{e}^{\mathrm{j} n \omega t} \mathrm{~d} t= \\
& \sum_{n=-\infty}^{+\infty} F_{1}(n \omega) \frac{1}{T} \int_{0}^{T} f_{2}(t) \mathrm{e}^{\mathrm{j} n \omega t} \mathrm{~d} t= \\
& \sum_{n=-\infty}^{+\infty} F_{1}(n \omega) \frac{1}{T} \int_{0}^{T} f_{2}(t) \overline{\mathrm{e}^{-\mathrm{j} n \omega t}} \mathrm{~d} t= \\
& \sum_{n=-\infty}^{+\infty} F_{1}(n \omega) \frac{1}{T} \int_{0}^{T} f_{2}(t) \mathrm{e}^{-\mathrm{j} n \omega t} \mathrm{~d} t= \\
& \sum_{n=-\infty}^{+\infty} F_{1}(n \omega) F_{2}(-n \omega) .
\end{aligned}
$$

If $f_{1}(t)=f_{2}(t)=f(t)$, then the above Property 4 turns to be the well-known discrete frequency-domain Parseval's energy equality as shown in [17] as

$$
\frac{1}{T} \int_{0}^{T}|f(t)|^{2} \mathrm{~d} t=\sum_{n=-\infty}^{+\infty}|F(n \omega)|^{2}=\frac{1}{4} \sum_{n=-\infty}^{+\infty} A_{n}^{2}
$$

where $|F(n \omega)|=\frac{1}{2} A_{n}, A_{n}$ is the magnitude of the sinusoidal and cosine waves with frequency $n \omega, n=$ 
$0, \pm 1, \pm 2, \cdots$. Thus, $A_{n}$ is called as the spectrum of the sinusoidal and cosine waves at the frequency $n \omega, n=$ $0, \pm 1, \pm 2, \cdots$.

From the above Parseval's energy equality (5), it is observed that the average energy of a signal in continuous domain can be expressed as a quarter of the summation of the square spectrums of all frequencies $n \omega, n=0, \pm 1, \pm 2, \cdots$, in discrete frequency domain. What follows is to adopt the discrete-frequency energy formula $\sum_{n=-\infty}^{+\infty}|F(n \omega)|^{2}$ for evaluating the tracking performance.

\section{Iterative learning control scheme}

Consider a class of linear time-invariant SISO systems taking the form as

$$
\left\{\begin{array}{l}
\dot{x}_{k}(t)=A x_{k}(t)+B u_{k}(t) \\
y_{k}(t)=C x_{k}(t), \quad t \in[0, T]
\end{array}\right.
$$

where $[0, T]$ is an operation time interval, the subscript $k$ refers to the operation number. $x_{k}(t) \in \mathbf{R}^{n}, u_{k}(t) \in \mathbf{R}$ and $y_{k}(t) \in \mathbf{R}$ denote an $n$-dimensional state vector, a scalar control input and an output at the $k$-th iteration, while $A, B$ and $C$ are matrices with appropriate dimensions. Further, assume that the relative degree $r$ of the system (6) is greater than unity $(r>1)$.

For system (6), the current proportional error and its $r$ th-order derivative are utilized to compensate for its control input so as to generate the next control input, which forms a $\mathrm{PD}^{(r)}$-type ILC scheme as follows:

$u_{1}(t)$ is given arbitrarily

$$
\begin{gathered}
u_{k+1}(t)=u_{k}(t)+\Gamma_{p} e_{k}(t)+\Gamma_{r} e_{k}^{(r)}(t), \quad t \in[0, T], \\
k=1,2,3 \cdots .
\end{gathered}
$$

Here, $\Gamma_{p}$ and $\Gamma_{r}$ are assigned as the proportional and the $r$-th-order derivative learning gains, respectively.

Applying Property 2 of Lemma 1 to both sides of (6), we get

$\left\{\begin{array}{l}{\left[\frac{1}{T}\left(x_{k}(T)-x_{k}(0)\right)\right]+\mathrm{j} n \omega X_{k}(n \omega)=A X_{k}(n \omega)+B U_{k}(n \omega)} \\ Y_{k}(n \omega)=C X_{k}(n \omega), \quad n=0, \pm 1, \pm 2, \cdots\end{array}\right.$

where

$$
\begin{aligned}
& X_{k}(n \omega)=\frac{1}{T} \int_{0}^{T} x_{k}(t) \mathrm{e}^{-\mathrm{j} n \omega t} \mathrm{~d} t \\
& U_{k}(n \omega)=\frac{1}{T} \int_{0}^{T} u_{k}(t) \mathrm{e}^{-\mathrm{j} n \omega t} \mathrm{~d} t \\
& Y_{k}(n \omega)=\frac{1}{T} \int_{0}^{T} y_{k}(t) \mathrm{e}^{-\mathrm{j} n \omega t} \mathrm{~d} t \\
& \omega=\frac{2 \pi}{T} .
\end{aligned}
$$

Further,

$$
\begin{aligned}
& X_{k}(n \omega)=(\mathrm{j} n \omega I-A)^{-1} B U_{k}(n \omega)- \\
& \quad(\mathrm{j} n \omega I-A)^{-1}\left[\frac{1}{T}\left(x_{k}(T)-x_{k}(0)\right)\right] .
\end{aligned}
$$

Thus,

$$
\begin{aligned}
& Y_{k}(n \omega)=C(\mathrm{j} n \omega I-A)^{-1} B U_{k}(n \omega)- \\
& C(\mathrm{j} n \omega I-A)^{-1}\left[\frac{1}{T}\left(x_{k}(T)-x_{k}(0)\right)\right] .
\end{aligned}
$$

Analogously, the $\mathrm{PD}^{(r)}$-type ILC law (7) leads to

$$
\begin{gathered}
U_{k+1}(n \omega)=U_{k}(n \omega)+\left(\Gamma_{p}+\Gamma_{r}(\mathrm{j} n \omega)^{r}\right) E_{k}(n \omega)+ \\
\frac{1}{T} \Gamma_{r} \sum_{i=0}^{r-1}(\mathrm{j} n \omega)^{r-1-i}\left(e_{k}^{(i)}(T)-e_{k}^{(i)}(0)\right)
\end{gathered}
$$

Here, $E_{k}(n \omega)=\frac{1}{T} \int_{0}^{T} e_{k}(t) \mathrm{e}^{-\mathrm{j} n \omega t} \mathrm{~d} t$.

Owing to

$$
e_{k+1}(t)=y_{d}(t)-y_{k+1}(t)=e_{k}(t)-\left(y_{k+1}(t)-y_{k}(t)\right)
$$

then,

$$
\begin{aligned}
& E_{k+1}(n \omega)=E_{k}(n \omega)-\left(Y_{k+1}(n \omega)-Y_{k}(n \omega)\right)= \\
& E_{k}(n \omega)-C(\mathrm{j} n \omega I-A)^{-1} B\left(U_{k+1}(n \omega)-U_{k}(n \omega)\right)+ \\
& C(\mathrm{j} n \omega I-A)^{-1} \times \frac{1}{T}\left[\left(x_{k+1}(T)-x_{k}(T)\right)-\right. \\
& \left.\quad\left(x_{k+1}(0)-x_{k}(0)\right)\right] .
\end{aligned}
$$

Substituting (8) into (9) reduces

$$
\begin{aligned}
& E_{k+1}(n \omega)=E_{k}(n \omega)- \\
& C(\mathrm{j} n \omega I-A)^{-1} B\left(\Gamma_{p}+\Gamma_{r}(\mathrm{j} n \omega)^{r}\right) E_{k}(n \omega)- \\
& C(\mathrm{j} n \omega I-A)^{-1} B \frac{\Gamma_{r}}{T} \sum_{i=0}^{r-1}(\mathrm{j} n \omega)^{r-1-i}\left(e_{k}^{(i)}(T)-e_{k}^{(i)}(0)\right)+ \\
& C(\mathrm{j} n \omega I-A)^{-1} \frac{1}{T}\left[\left(x_{k+1}(T)-x_{k}(T)\right)-\right. \\
& \left.\left(x_{k+1}(0)-x_{k}(0)\right)\right] .
\end{aligned}
$$

Let

$$
\begin{aligned}
& G_{P D^{(r)}}(n \omega)=1-C(\mathrm{j} n \omega I-A)^{-1} B\left(\Gamma_{p}+\Gamma_{r}(\mathrm{j} n \omega)^{r}\right) \\
& \psi_{k}(n \omega)=\frac{1}{T} \sum_{i=1}^{r-1}(\mathrm{j} n \omega)^{r-1-i}\left(e_{k}^{(i)}(T)-e_{k}^{(i)}(0)\right) \\
& \Phi_{k}(0)=\frac{1}{T}\left(x_{k+1}(0)-x_{k}(0)-(\mathrm{j} n \omega)^{r-1} \Gamma_{r} B e_{k}(0)\right) \\
& \Phi_{k}(T)=\frac{1}{T}\left(x_{k+1}(T)-x_{k}(T)-(\mathrm{j} n \omega)^{r-1} \Gamma_{r} B e_{k}(T)\right) .
\end{aligned}
$$

Arranging (10), we get

$$
\begin{aligned}
& E_{k+1}(n \omega)=G_{P D^{(r)}}(n \omega) E_{k}(n \omega)- \\
& \quad C(\mathrm{j} n \omega I-A)^{-1} B \Gamma_{r} \psi_{k}(n \omega)+C(\mathrm{j} n \omega I-A)^{-1} \times \\
& \quad\left(\Phi_{k}(\mathrm{~T})-\Phi_{k}(0)\right) .
\end{aligned}
$$

Remark 1. It is found from (11) that, for the $\mathrm{PD}^{(r)}$-type ILC law (7), the spectrum of the tracking error at the next iteration is composed of three parts. The first part is the spectrum of the current tracking error which dominates the convergence. The second part includes the values at the 
current iteration including the initial and terminal states as well as the initial and terminal tracking error derivatives whose orders range from zero to $r-1$. The third part consists of the values at the next iteration of the initial and terminal states.

\section{Convergence analysis}

Theorem 1. Assume that the $\mathrm{PD}^{(r)}$-type ILC (7) is applied to the linear time-invariant systems (6) with relative degree $r>1$. Suppose $e_{k}^{(i)}(T)=0, e_{k}^{(i)}(0)=0$, for all $i=0,1,2, \cdots, r-1$, and the initial and terminal states are resectable. Then, the propositions

$$
\sum_{n=-\infty}^{+\infty}\left|E_{k+1}(n \omega)\right|^{2}<\sum_{n=-\infty}^{+\infty}\left|E_{k}(n \omega)\right|^{2}
$$

and

$$
\lim _{k \rightarrow+\infty} \sum_{n=-\infty}^{+\infty}\left|E_{k+1}(n \omega)\right|^{2}=0
$$

hold if and only if

$$
\left|G_{\mathrm{PD}^{(r)}}(n \omega)\right|<1
$$

where

$$
\left|G_{\mathrm{PD}^{(r)}}(n \omega)\right|=\left|1-C(\mathrm{j} n \omega I-A)^{-1} B\left(\Gamma_{p}+\Gamma_{r}(\mathrm{j} n \omega)^{r}\right)\right| .
$$

Proof. Sufficiency:

Because $e_{k}^{(i)}(T)=0$ and $e_{k}^{(i)}(0)=0$, for $i=$ $0,1,2, \cdots, r-1$, and the initial and terminal states are resectable, (11) becomes

$$
E_{k+1}(n \omega)=G_{\mathrm{PD}^{(r)}}(n \omega) E_{k}(n \omega) .
$$

Denote $Q_{k}=\sum_{n=-\infty}^{+\infty}\left|E_{k}(n \omega)\right|^{2}$. By taking the assumption $\left|G_{\mathrm{PD}^{(r)}(n \omega)}\right|<1$ into account, it is induced that $0 \leq Q_{k+1}<Q_{k}<\cdots<Q_{1}$. This means that the sequence $\left\{Q_{k+1}\right\}$ is lower-bounded and monotonically decreasing. Therefore, $\lim _{k \rightarrow+\infty} Q_{k+1}$ exists.

What follows is to prove that $\lim _{k \rightarrow+\infty} Q_{k+1}=0$ by reduction to absurdity.

Suppose that $\lim _{k \rightarrow+\infty} Q_{k+1}=Q>0$. Then, for the given constant $\varepsilon_{1}=\frac{Q}{2}$, there exists such a positive integer $K_{0}$ that, for all $k>K_{0}$, the inequality $Q_{k+1}>Q-\varepsilon_{1}=\frac{Q}{2}$ holds.

Recall that the series $Q_{k+1}=\sum_{n=-\infty}^{+\infty}\left|E_{k+1}(n \omega)\right|^{2}$ is convergent. This means that, for all $k>K_{0}$, the limit exists for the partial summation sequence $Q_{k+1}(m)=$ $\sum_{n=-m}^{+m}\left|E_{k+1}(n \omega)\right|^{2}$ with respect to the index $m$. In specific, $\lim _{m \rightarrow \infty} Q_{k+1}(m)=Q_{k+1}$. This implies that, for the given constant $\varepsilon_{2}=\frac{Q}{4}$, there exists a positive integer $N_{0}$ so that, for all $m>N_{0}$, the inequality

$Q_{k+1}(m)=\sum_{n=-m}^{+m}\left|E_{k+1}(n \omega)\right|^{2}>Q_{k+1}-\varepsilon_{2}>Q-\varepsilon_{1}-\varepsilon_{2}$ is true.
In particular, it yields that

$$
Q_{k+1}\left(2 N_{0}\right)=\sum_{n=-2 N_{0}}^{+2 N_{0}}\left|E_{k+1}(n \omega)\right|^{2}>Q-\varepsilon_{1}-\varepsilon_{2} .
$$

As the total number of the terms in the $Q_{k+1}\left(2 N_{0}\right)$ formulation is equal to $4 N_{0}+1$, the inequality (16) implies that there at least exists an integer $n_{0}$ so that $-2 N_{0} \leq n_{0} \leq 2 N_{0}$ and

$$
\left|E_{k+1}\left(n_{0} \omega\right)\right|^{2}>\frac{Q-\varepsilon_{1}-\varepsilon_{2}}{4 N_{0}+1}=\frac{Q}{4\left(4 N_{0}+1\right)}>0 .
$$

On the other hand, (15) reduces to

$$
E_{k+1}\left(n_{0} \omega\right)=\left(G_{\mathrm{PD}^{(r)}}\left(n_{0} \omega\right)\right)^{k} E_{1}\left(n_{0} \omega\right) .
$$

By considering the assumption $\left|G_{\mathrm{PD}^{(r)}}\left(n_{0} \omega\right)\right|<1$, it results in

$$
\lim _{k \rightarrow \infty} E_{k+1}\left(n_{0} \omega\right)=0 .
$$

This is contradictory to the conclusion (17).

The contradiction leads that the propositions (12) and (13) are true.

This completes the proof of sufficiency.

Necessity:

Assume that (14) does not always hold. Then, there exists at least such a number $n_{0}$ that

$$
\left|G_{\mathrm{PD}^{(r)}}\left(n_{0} \omega\right)\right| \geq 1 .
$$

The equality (15) leads to

$$
\left|E_{k+1}\left(n_{0} \omega\right)\right|=\left|G_{\mathrm{PD}^{(r)}}\left(n_{0} \omega\right)\right|^{k}\left|E_{1}\left(n_{0} \omega\right)\right| \geq\left|E_{1}\left(n_{0} \omega\right)\right| .
$$

Consequently,

$$
\sum_{n=-\infty}^{+\infty}\left|E_{k+1}(n \omega)\right|^{2} \geq\left|E_{k+1}\left(n_{0} \omega\right)\right|^{2} \geq\left|E_{1}\left(n_{0} \omega\right)\right|^{2}
$$

and

$$
\lim _{k \rightarrow \infty} \sum_{n=-\infty}^{+\infty}\left|E_{k+1}(n \omega)\right|^{2} \geq \lim _{k \rightarrow \infty}\left|E_{1}\left(n_{0} \omega\right)\right|^{2}=\left|E_{1}\left(n_{0} \omega\right)\right|^{2} .
$$

It is possible to select $U_{1}(n \omega)$ and $Y_{d}(n \omega)$ such that $\left|E_{1}\left(n_{0} \omega\right)\right|>0$, which contradicts to the postulate (12).

This completes the proof of necessity.

Remark 2. In Theorem 1 the sufficient and necessary assumptions for the monotone convergence require that not only all of the initial states and tracking errors are resectable, but also all of the terminal states and the tracking errors are resectable. It is well-known that, up to this date, in the existing ILC investigations for guaranteeing the convergence, the requirement for the resetting of the initial states and tracking errors, has been accepted in ILC community. But the requirement for the resetting of the terminal states and tracking errors is new. Thus, the convergent assumptions in Theorem 1 seem to convey that, in discrete frequency domain, the assumptions are quite critical and 
different from the existing results in continuous-time domain. However, the assumptions are possibly satisfied for a stable system without steady-state error.

Without involving the reset assumptions, it is found that, in discrete frequency domain, the recursive spectrums relationship $E_{k+1}(n \omega)=G_{\mathrm{PD}^{(r)}}(n \omega) E_{k}(n \omega)$ of two adjacent iterations is directly acquired in a precise equality form rather than the time-domain relationship $\left\|e_{k+1}(\cdot)\right\|<\tilde{\rho}\left\|e_{k}(\cdot)\right\|$ in an inequality form by an appropriate relaxation technique. The discrepancy perhaps delivers that the sufficient assumption (14) in discrete frequency domain is more efficient than that of possibly achieved in continuous time domain. We guess that it is the milder convergent condition (14) that incurs the additional requirement for the resetting of the terminal states and the tracking errors. However, the conjectures need to be clarified in a rigorous manner.

Remark 3. From the formulation of the proportional$r$-th-order derivative-type ILC scheme (7), it is seen that the scheme will be degenerated to a proportional-type ILC scheme when the $r$-th-order derivative learning gain $\Gamma_{r}$ is null. Then, under the assumption of the initial and terminal resetting, it is not difficult to deduce that the sufficient and necessary condition for guaranteeing the monotone convergence becomes

$$
\left|G_{\mathrm{P}}(n \omega)\right|=\left|1-C(\mathrm{j} n \omega I-A)^{-1} B \Gamma_{p}\right|<1 .
$$

\section{Numerical simulations}

To show the effectiveness of the learning control law (7) in discrete frequency domain, consider an SISO linear timeinvariant system described as

$$
\left\{\begin{array}{l}
{\left[\begin{array}{l}
\dot{x}_{1}(t) \\
\dot{x}_{2}(t)
\end{array}\right]=\left[\begin{array}{cc}
0 & 1 \\
-\frac{1}{3} & -\frac{1}{4}
\end{array}\right]\left[\begin{array}{l}
x_{1}(t) \\
x_{2}(t)
\end{array}\right]+\left[\begin{array}{l}
0 \\
1
\end{array}\right] u(t)} \\
y(t)=\left[\begin{array}{ll}
\frac{1}{3} & 0
\end{array}\right]\left[\begin{array}{l}
x_{1}(t) \\
x_{2}(t)
\end{array}\right] \\
x(0)=\left[\begin{array}{ll}
x_{1}(0) & x_{2}(0)
\end{array}\right]^{\mathrm{T}}=\left[\begin{array}{ll}
0 & 0
\end{array}\right]^{\mathrm{T}}
\end{array}\right.
$$

It is testified that $C B=0$ and $C A B=\frac{1}{3} \neq 0$. This means that the relative degree of system (18) is equal to 2 , i.e., $r=2$. The operation time interval of system (18) is set as $[0,40]$. The desired trajectory is chosen as $y_{d}(t)=$ $1-\mathrm{e}^{-0.25 t^{2}}$ and the beginning control input is chosen as $u_{1}(t)=1$. As $x_{k}(0)=0$, it is obvious that $e_{k}(0)=0$ and $e_{k}^{(1)}(0)=0$. By solving (18), it is easy to testify that the system (18) is stable with no steady-state error for steptype inputs. This implies that the resetting of the terminal states and tracking errors is inherently guaranteed. For the $\mathrm{PD}^{(2)}$-type ILC scheme (7), the learning gains are selected as $\Gamma_{p}=0.8$ and $\Gamma_{2}=2.4$. It is computed that

$$
\left|G_{\mathrm{PD}^{(2)}}(n \omega)\right|=\left(\frac{5.76 n^{4} \omega^{4}-5.16 n^{2} \omega^{2}+0.64}{144 n^{4} \omega^{4}-87 n^{2} \omega^{2}+16}\right)^{\frac{1}{2}}<1
$$

where $\omega=\frac{2 \pi}{40} \approx 0.157$, which means the convergent condition $\left|G_{\mathrm{PD}^{(r)}}(n \omega)\right|<1$ holds. Fig. 1 displays the tracking performance of the output at the 2 nd and 15 th implementations.

Fig. 2 depicts the spectrums of the frequency-wise tracking errors in the discrete frequency domain. It is seen that the tracking error spectrum at each fixed frequency is monotonously decreasing in the iteration direction. The monotone convergence related to the spectrums of the tracking errors made by the $\mathrm{PD}^{(r)}$-type ILC law (7) is illustrated in Fig. 3 and the monotonicity in terms of the average power of the tracking error is manifested in Fig. 4, respectively.

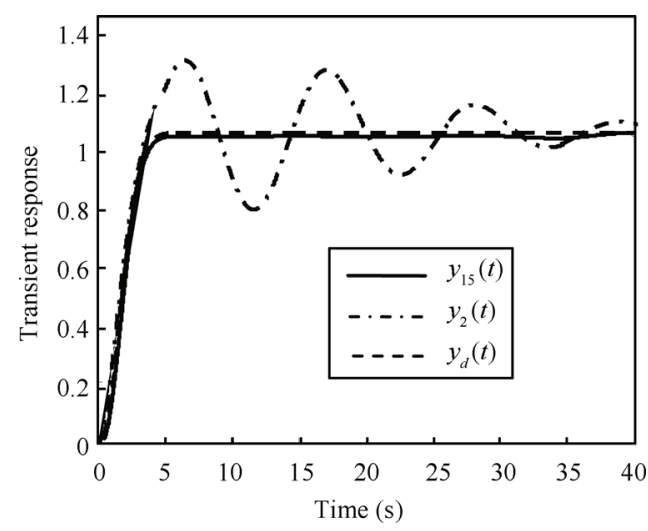

Fig. 1 Outputs at the 2nd and 15th iterations

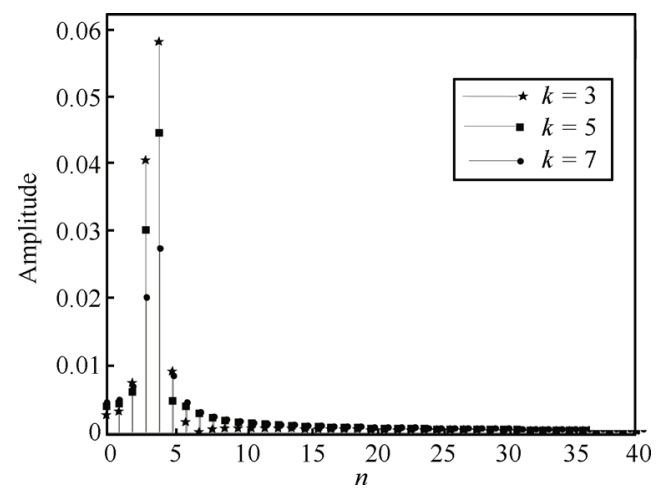

Fig. 2 Spectrums at the 3 rd, 5 th and 7 th iterations

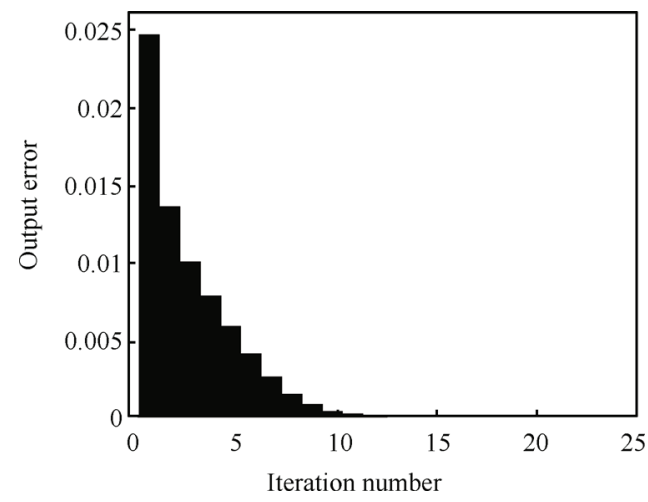

Fig. 3 Tracking error tendency in discrete-frequency domain 


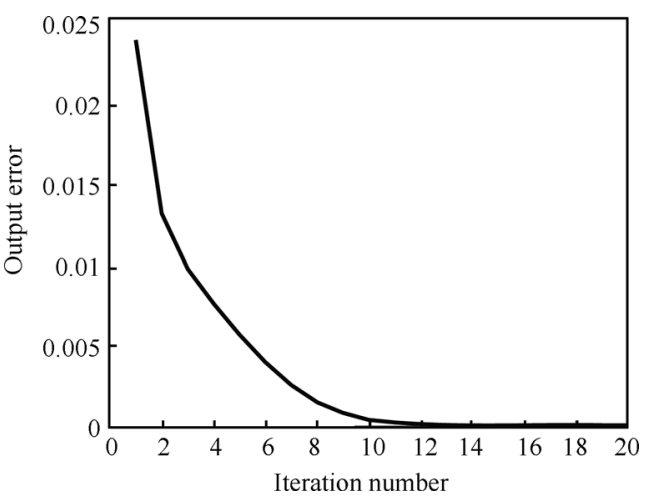

Fig. 4 Tracking error tendency in continuous-time domain

\section{Conclusions}

In this paper, for a class of linear time-invariant systems with relative degree $r>1$, a $\mathrm{PD}^{(r)}$-type ILC law is developed and its sufficiency and necessity for monotone convergence is analyzed by means of evaluating the tracking error in Parseval's energy form in discrete frequency domain. For analysis, the properties of Fourier coefficients regarding the system dynamics as well as the proposed ILC algorithm are discussed. In discrete frequency domain, the mathematical representation of signals, initial and terminal assumptions and the recursive relationship mode of the tracking errors between two adjacent operations are quite different from those of continuous time domain, and as such, the sufficiently convergent equivalence of the two domains is not obvious. This type of discrepancy needs to be further clarified in the future. In addition, the perturbation, noise as well as system parametric uncertainties are unavoidable in real applications. Therefore, robustness of the proposed ILC scheme to these perturbations, e.g., load and measurement perturbations, sensitivity due to the higherorder derivation computation, remains a challenging issue. It will be addressed in future.

\section{References}

[1] S. Arimoto, S. Kawamura, F. Miyazaki. Bettering operation of robots by learning. Journal of Robotic Systems, vol.1, no. 2 , pp. $123-140,1984$.

[2] K. L. Moore. Iterative learning control: An expository overview. Applied and Computational Control, Signals, and Circuits, vol.1, pp. 151-214, 1999.

[3] Y. Q. Chen, K. L. Moore. A practical iterative learning path-following control of an omni-directional vehicle. Asian Journal of Control, vol. 4, no. 1, pp. 90-98, 2002.

[4] Z. Bien, K. M. Huh. Higher-order iterative learning control algorithm. IEE Proceedings: Control Theory and Applications, vol. 136, no. 3, pp. 105-112, 1989.

[5] K. L. Moore, J. X. Xu. Special issue on iterative learning control. International Journal of Control, vol. 73, no. 10, pp. 819-823, 2000.
[6] X. E. Ruan, Z. Z. Bien, Q. Wang. Convergence properties of iterative learning control processes in the sense of the Lebesgue- $p$ norm. Asian Journal of Control, vol. 14, no. 4 pp. 1095-1107, 2012.

[7] M. X. Sun, D. W. Wang, G. Y. Xu. Initial shift problem and its ILC solution for nonlinear systems with higher relative degree. In Proceedings of the American Control Conference, IEEE, Chicago, USA, vol. 1, no. 6, pp. 277-281, 2000.

[8] Z. Q. Song, J. Q. Mao, S. W. Dai. First-order D-type iterative learning control for nonlinear systems with unknown relative degree. Acta Automatica Sinica, vol. 31, no. 4, pp. 555-561, 2006.

[9] M. X. Sun, D. W. Wang, G. Y. Xu. Sampled-data iterative learning control for SISO nonlinear systems with arbitrary relative degree. In Proceedings of the American Control Conference, vol. 1, no. 6, pp.667-671, 2000.

[10] M. X. Sun, D. W. Wang. Anticipatory iterative learning control for nonlinear systems with arbitrary relative degree. IEEE Transactions on Automatic Control, vol.46, no. 5 , pp. 783-788, 2001.

[11] X. E. Ruan, Q. Wang, J. Wang. Convergence property of relative degree-based iterative learning control in the sense of Lebesgue- $p$ norm. In Proceedings of the 30th Chinese Control Conference, IEEE, Yantai, China, pp. 2446-2449, 2011.

[12] D. W. Wang, Y. Q. Ye. Design and experiments of anticipatory learning control: Frequency-domain approach IEEE/ASME Transactions on Mechatronics, vol.10, no. 3, pp. 305-313, 2005.

[13] H. S. Li, Y. Q. Chen, J. H. Zhang, X. L. Wen. A tuning algorithm of PD-type iterative learning control. In Proceedings of the Chinese Control and Decision Conference, IEEE, Xuzhou, China, pp. 1-6, 2010.

[14] D. Y. Meng, Y. M. Jia, J. P. Du, F. S. Yu. Frequencydomain approach to robust iterative learning controller design for uncertain time-delay systems. In Proceedings of the Joint 48th IEEE Conference on Decision and 28th Chinese Control Conference, IEEE, Shanghai, China, pp. 4870$4875,2009$.

[15] A. Isidori. Nonlinear Control Systems, London, UK Springer-Verlag, 1995.

[16] A. Pinkus. Fourier Series and Integral Transforms, Cambridge, UK: Cambridge University Press, 1997.

[17] S. Salivahanan, A. Vallavaraj, C. Gnanaapriya. Digital Signal Processing, New Delhi, India: McGraw-Hill, 2000.

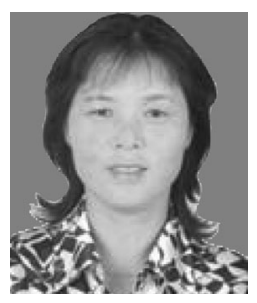

Xiao-E Ruan received the B.Sc. and M.Sc. degrees in pure mathematics education from Shaanxi Normal University, China in 1988 and 1995, respectively. She received the $\mathrm{Ph}$. D. degree in control science and engineering from $\mathrm{Xi}^{\prime}$ an Jiaotong University, China in 2002. From March 2003 to August 2004, she worked as a post-doctoral fellow at the Department of Electrical Engineering, Korea Advance Institute of Science and Technology, 
Korea. From September 2009 to August 2010, she worked as a visiting scholar at Ulsan National Institute of Science and Technology, Korea. Since 1995, she joined in Xi'an Jiaotong University. Currently, she is a full professor in School of Mathematics and Statistics. She has published more than 40 academic papers.

Her research interests include iterative learning control and optimized control for large-scale systems.

E-mail: wruanxe@mail.xjtu.edu.cn (Corresponding author)

ORCID iD: 0000-0003-3049-4075

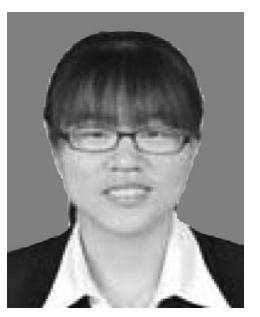

Zhao-Zhen Li received the B. Sc. degree in mathematical from Inner Mongolia Normal University China in 2011. Currently, she is a graduate student of $\mathrm{Xi}^{\prime}$ an Jiaotong University, China.

Her research interest is iterative learning control.

E-mail: lzz0427@stu.xjtu.edu.cn

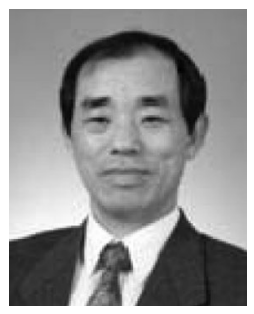

Z. Z. Bien received the B. Sc. degree in electronic engineering from Seoul National University of Korea, Korea in 1969, the M. Sc. and Ph. D. degrees in electrical engineering from University of Iowa, USA in 1972 and 1975 respectively. From 1977 to 2009, he has been with the Department of Electrical Engineering and Computer Science, Korea Advanced Institute of Science and Technology of Korea, Korea. From March 2009 to February 2014, he was a chair professor in the Department of Electrical and Computer Engineering, Ulsan National Institute of Science and Technology, Korea. Since 2007, he has been awarded as IEEE Fellow and International Federation of Fuzzy Association (IFSA) Fuzzy Fellow. Also, he has been an academician of Korea Institute of Science and Technology and an academician of Korea Institute of Engineering. He is a meritorious professor in the Department of Electrical Engineering and Computer Science, Korea Advanced Institute of Science and Technology, Korea.

His research interests include intelligent automation and learning control methods, soft-computing techniques with emphasis on fuzzy logic systems, assistive robots and human-robot interaction systems.

E-mail: zbien@ee.kaist.ac.kr 